\title{
Infrared Femtosecond Laser Preionization in Secondary Ion Mass Spectrometry of Silver Surface
}

\author{
Dusan Lorenc, ${ }^{1}$ Eduard Jane, ${ }^{2}$ Monika Stupavska, ${ }^{2}$ Monika Jerigova, ${ }^{1,2}$ Dusan Velic ${ }^{1,2}$ \\ ${ }^{1}$ International Laser Centre, Ilkovicova 3, 84104 Bratislava, Slovakia \\ ${ }^{2}$ Faculty of Natural Sciences, Comenius University, Mlynska dolina, 84215 Bratislava, Slovakia
}

\begin{abstract}
An alternative secondary ion mass spectrometry utilizing laser preionization is introduced. The native $\mathrm{Ag}$ sample surface is first irradiated with laser pulse (100 fs duration, $10^{10}-10^{11} \mathrm{~W} / \mathrm{cm}^{2}$ intensity, $1240 \mathrm{~nm}$ wavelength) and subsequently bombarded with primary ions $\left(\mathrm{Bi}_{3}{ }^{+}, 10 \mathrm{~ns}\right.$ duration, $25 \mathrm{keV}$ energy). Multiple correlation patterns are observed in the mass spectra, confirming the mutual laser-secondary ion mass spectrometry (SIMS) interplay in the preionization mechanism. The $\mathrm{Ag}^{+}, \mathrm{C}_{3} \mathrm{H}_{5}{ }^{+}, \mathrm{C}_{3} \mathrm{H}_{5} \mathrm{O}_{3}{ }^{+}$, and $\mathrm{AgOH}^{+}, \mathrm{C}_{4} \mathrm{H}_{5} \mathrm{O}_{4}{ }^{+}$are observed with the shallow and steep increasing of intensities at $1.3 \times 10^{11} \mathrm{~W} / \mathrm{cm}^{2}$ and $1.5 \times 10^{11} \mathrm{~W} / \mathrm{cm}^{2}$, respectively. Two ionization mechanisms are identified, the ion sputtering regime for intensities of less than $1.4 \times 10^{11} \mathrm{~W} / \mathrm{cm}^{2}$ and the multiphoton ionization at higher intensities. The $\mathrm{Ag}$ saturation intensity obtained from fitting is $2.4 \times 10^{13} \mathrm{~W} / \mathrm{cm}^{2}$, close to the one reported for postionization. The proposed preionization approach might eliminate the need for high peak power/high intensity laser source and, moreover, the experiment geometry ensures that large areas of the sample are affected by the laser beam.
\end{abstract}

Key words: fs Laser, SIMS, Preionization, Postionization, Matrix effect, Multiphoton ionization

\section{Introduction}

S econdary Ion Mass Spectrometry (SIMS) [1-4] has been S established as an extremely valuable tool providing comprehensive chemical and spatial analysis of a broad range of materials. However, the most relevant drawback of the SIMS technique is the matrix effect [4] turning the quantitative analysis into a very difficult procedure. The yield of specific secondary ions is strongly dependent on the chemical environment from which they are emitted and the variations might be of the order of several magnitudes. A possible approach to solve the problem is secondary neutral

Electronic supplementary material The online version of this article (doi:10.1007/s13361-012-0390-2) contains supplementary material, which is available to authorized users.

Correspondence to: Dusan Lorenc; e-mail: lorenc@ilc.sk mass spectrometry (SNMS). As opposed to the case of conventional SIMS, SNMS is based on sputtered neutrals allowing a direct quantitative analysis. While in the case of SIMS the secondary ions originate from the emission dynamics at the surface, SNMS uses an external source to postionize the sputtered neutral species. Most of the previously published results on laser postionization in the SNMS regime [5-14] were performed using either nanosecond (ns) UV lasers or femtosecond (fs) laser systems providing $800 \mathrm{~nm}$ or shorter wavelengths. However, the fs postionization regime requires intensities of the order of $10^{12}-10^{14} \mathrm{~W} / \mathrm{cm}^{2}$ (i.e., to enter the multiphoton ionization (MPI) domain, which in turn means that $\mathrm{mJ}$ class fs laser systems are required. Even though important improvements were made within the last decade, those systems still remain complex, bulky, and expensive.

A different approach to suppress the matrix effect and related issues is proposed within the scope of this work. 
Instead of postionization of the neutral species, the sample is a priori irradiated by fs laser beam with moderate intensity of the order of $10^{10}-10^{11} \mathrm{~W} / \mathrm{cm}^{2}$. The idea is to preionize the surface for the subsequent sputtering by the primary ions. The general arrangement resembles another method, matrixassisted laser desorption/ionization (MALDI) [15] but the preionization is matrix independent and (besides primary ions) uses an ultrafast (fs) IR laser source instead of the "slow" (ns) UV source generally used in MALDI.

\section{Experimental}

The MS part of the setup employed the TOF (time-of-flight) SIMS IV (ION-TOF GmbH, Germany). The sample surface was bombarded with $25 \mathrm{keV} \mathrm{Bi}_{3}{ }^{+}$primary ions with the pulse duration of $10 \mathrm{~ns}$ and the repetition rate of $50 \mathrm{~Hz}$. The MS were sampled using digital oscilloscope (LeCroy Wavesurfer 422, LeCroy Corp., Chestnut Ridge.(NY), U.S.) and subsequently downloaded for further processing, averaging 200 spectra for each scan.

A Cr:forsterite fs IR MOPA (Master Oscillator Power Amplifier) was used for preionization including a three-stage amplifier (100 fs, $4.5 \mathrm{~mJ}, 1240 \mathrm{~nm}$, photon energy $\sim 1 \mathrm{eV}$ ). The system was operated at the rate of $50 \mathrm{~Hz}$ and a set of delay generators (DG 535, SRS Inc., Sunnyvale (CA), U.S.) was used to synchronize the laser with SIMS. Note that the temporal resolution of the experiment is currently limited mainly by the mutual temporal jitter laser-SIMS being of the order of $1 \mathrm{~ns}$ while SIMS itself reportedly shows intrinsic temporal jitter of the order of $0.1 \mathrm{~ns}$ [personal communication].

The silver sample (Sigma-Aldrich, s.r.o., Praha, Czech Republic) in the form of thin plate was cleaned with acetone and placed in the off-focus geometry behind the beam waist in the divergent part of the laser beam. The geometry "first focus then sample" is advocated by the fact that the beam is focused into high vacuum and is thus free of any nonlinear/plasma effects that would arise at higher pressures. The optical setup allowed lowering the peak intensity at the sample surface while maintaining the flexibility of changing the beam spot size by moving the focusing lens ( $(\mathrm{f} 300)$. Good care was taken in order to avoid plasma formation at the sample surface during the experiments. Current experimental setup employs grazing incidence arrangement (i.e., the laser beam is impingent on the sample at low angle of approximately $3.5^{\circ}$. Such arrangement effectively maps the approximately circular cross-section of the laser beam to an elongated ellipse on the surface.

\section{Results and Discussion}

Three different experiments were compared. First, a control experiment was performed as standard static SIMS spectra of Ag surface. Second, the laser irradiation was used as the exclusive source of secondary ions from the $\mathrm{Ag}$ surface without bombardment by primary ions. The maximum achievable pulse energy of $3.6 \mathrm{~mJ}$ was set and the lens was then moved out of focus to prevent plasma formation at the sample surface. The laser pulse energy was then gradually lowered by a variable attenuator and MS were recorded at each point (Online Resource 1). Third, the laser irradiation was combined with SIMS sputtering and the corresponding data are shown in Figure 1. Multiple fine MS features arise as a result of mutual interplay between the fs laser and SIMS (Online Resource 2). To better visualize the differences, the data (data matrix) for SIMS sputtering and the data corresponding to the laser ionization were subtracted from the preionization data. The obtained spectral features corresponding to the laser-SIMS interplay are marked in dotted regions in Figure 1. Interestingly, the spectral peak of $\mathrm{Ag}^{+}$remains almost unaffected by the preionization procedure. The preionization induced secondary ions of higher masses in the range of 170-200 u correspond to organic fragments $\mathrm{C}_{\mathrm{x}} \mathrm{H}_{\mathrm{y}} \mathrm{O}_{\mathrm{z}}$ and/or $\mathrm{C}_{\mathrm{x}} \mathrm{H}_{\mathrm{y}}$ associated with $\mathrm{Ag}^{+}$as $\mathrm{C}_{\mathrm{x}} \mathrm{H}_{\mathrm{y}} \mathrm{O}_{\mathrm{z}} \mathrm{Ag}^{+} / \mathrm{C}_{\mathrm{x}} \mathrm{H}_{\mathrm{y}} \mathrm{Ag}^{+}$originating from native surface composition after only basic cleaning. The alternative explanation by double-charge secondary ions is improbable since the obtained MS were dominated by single charge molecular and fragment ion distributions. Moreover, $\mathrm{Ag}^{2+}$ secondary ions were not observed in any of MS. Note that this study had no direct intention to explicitly identify the higher mass ions and/or higher charges; rather it explores the differences resulting from laser-SIMS interplay. Some of the more prominent peaks, identified in MS, will be discussed below.

In order to quantitatively confirm that the resulting spectrum is not a trivial linear superposition of laser ionization and ion sputtering, function optimization algorithm was applied (Online Resource 3) with the results shown in Figure 2. Comparison with the experimental MS of preionization in Figure 1 and the calculated residuals imply that the resulting spectrum is the outcome of nontrivial laserSIMS interplay. Some of the previous works (e.g., $[16,17])$ have indicated that the temperature of the sample would be an important parameter affecting the sputtering process. The influence of the sample temperature was evaluated in a separate experiment, with the results shown in the inset of Figure 2. The sample was fixed to a thermoelectrically heated holder and the temperature was increased in steps from $25^{\circ}$ to $300^{\circ}$. The laser pulse energy was kept at the lower end of the range $(1.8 \mathrm{~mJ})$ during the experiment. Negligible differences in the MS are observed over the whole range of temperatures. Further, since the laser system is operated at $50 \mathrm{~Hz}$ with average power of less then $200 \mathrm{~mW}$, a very limited amount of energy is deposited on the sample. In fact, while measuring the temperature in situ on the sample surface (with a contact probe) during the preionization experiment, a temperature increase of only $4^{\circ}$ was observed over the period of a typical experimental run.

There are noticeable differences in the energy/intensity dependence of the peaks identified in the Figure 1, and to better resolve and to quantify intensity dependent changes in the MS, the integrated values of average ion signal are 


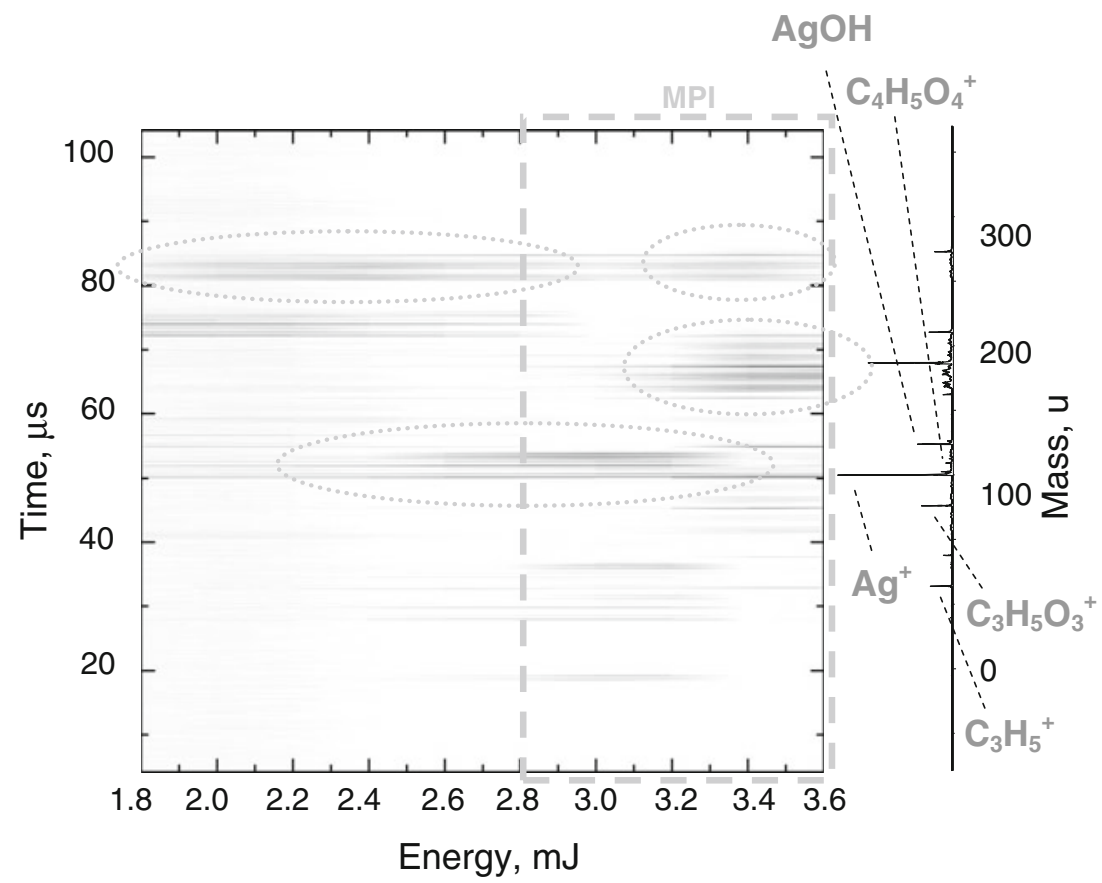

Figure 1. Energy scaling of MS in pre-ionization of Ag. Laser-SIMS interplay (correlation) patterns in pre-ionization MS are marked as dotted regions

shown in Figure 3 in a log-log plot. Clearly $\mathrm{Ag}^{+}, \mathrm{C}_{3} \mathrm{H}_{5}{ }^{+}$, and $\mathrm{C}_{3} \mathrm{H}_{5} \mathrm{O}_{3}{ }^{+}$all show qualitatively similar dependence with monotonic increase of ion signal eventually resulting into saturation at the higher intensities of $1.7-1.9 \times 10^{11} \mathrm{~W} / \mathrm{cm}^{2}$. A significantly different dependence is observed in the case of $\mathrm{AgOH}^{+}$and $\mathrm{C}_{4} \mathrm{H}_{5} \mathrm{O}_{4}{ }^{+}$. The ion signal of $\mathrm{AgOH}^{+}$initially

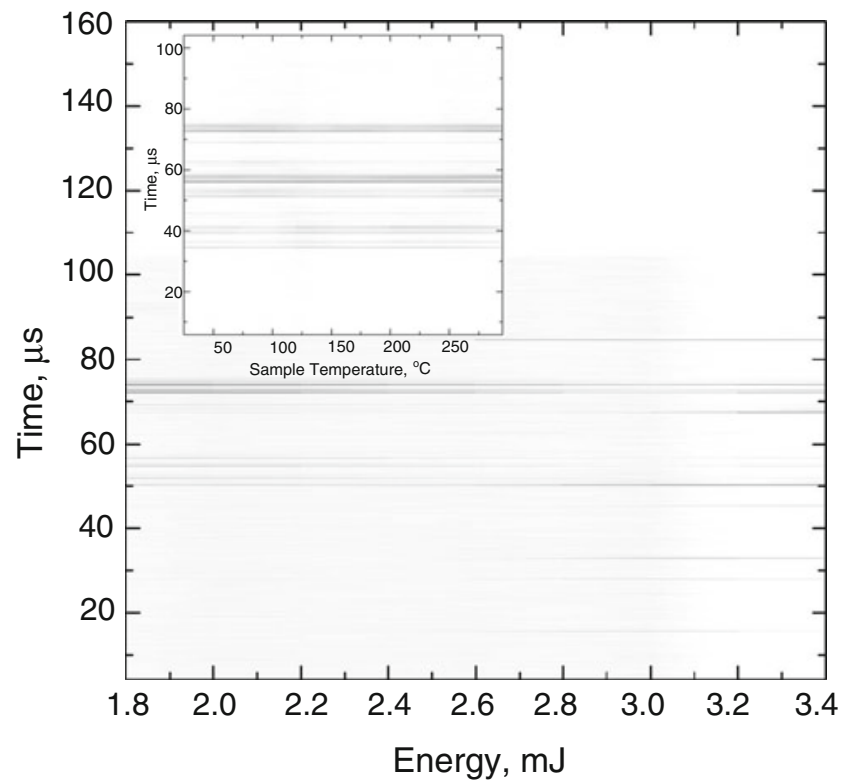

Figure 2. Energy scaling of MS as obtained by the optimization procedure assuming a trivial superposition of ion sputtering and laser ionization. The temperature-dependent MS obtained for laser pulse energy fixed to $1.8 \mathrm{~mJ}$ (inset) drops to a local minimum followed by a steep rise and eventual saturation. On the other hand, the $\mathrm{C}_{4} \mathrm{H}_{5} \mathrm{O}_{4}{ }^{+}$ion signal first reaches a local maximum with a subsequent fast drop-off. Both of the latter cases are an exclusive feature of preionization (i.e., laser-SIMS interplay and are not observed for the direct ionization by the laser. Moreover, a certain anticorrelation (complementarity) between the data for $\mathrm{AgOH}^{+}$and $\mathrm{C}_{4} \mathrm{H}_{5} \mathrm{O}_{4}{ }^{+}$is observed. The observed variations of ion signal might be explained as follows. At

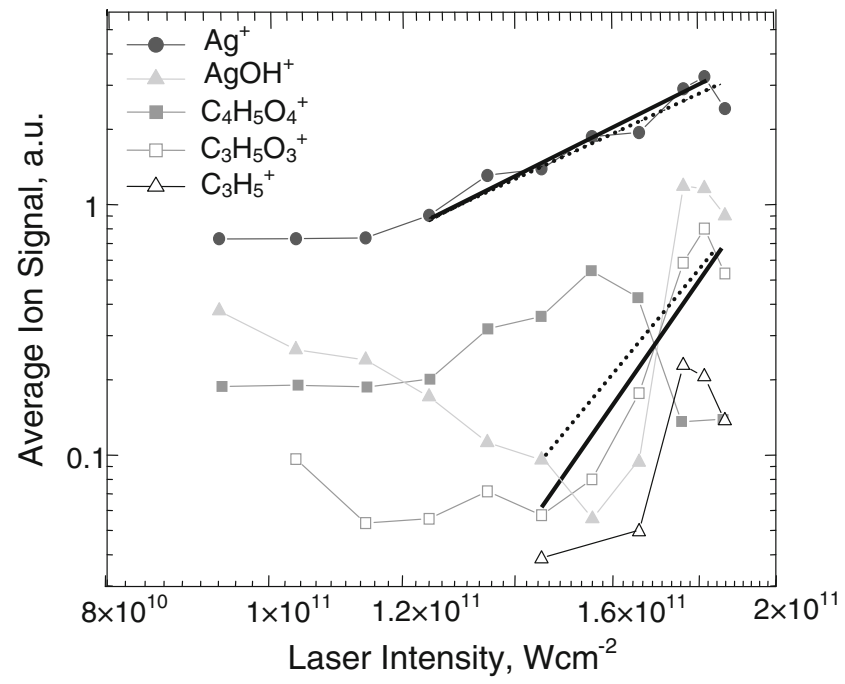

Figure 3. Average ion signal of selected peaks in the preionization spectra as a function of laser pulse peak intensity at the sample. Bold lines are theoretical fits by (Equation 1) [solid] and (Equation 2) [dotted], thin lines are only to guide the eye 
low laser intensities, the ion sputtering remains the dominant ionization mechanism, providing approximately constant number of secondary ions for a specific fragment. As the intensity increases, the competing processes of laser ionization and fragmentation come into play. At a further increase of laser intensity, the ionization enters the MPI domain and fragments are quickly ionized up to a level of saturation. The competing channels of ionization and fragmentation mean that (e.g., for $\mathrm{C}_{4} \mathrm{H}_{5} \mathrm{O}_{4}{ }^{+}$) a certain maximum ion yield is reached at $1.7-1.9 \times 10^{11} \mathrm{~W} / \mathrm{cm}^{2}$ before strong fragmentation takes place and the ion yield is suppressed. Although the change is not that abrupt in the case of $\mathrm{AgOH}^{+}$, the ion signal is systematically depleted by the interaction with the fs laser pulses and other ionization channels (e.g., intensity of $\mathrm{C}_{4} \mathrm{H}_{5} \mathrm{O}_{4}{ }^{+}$secondary ions is enhanced at the same time).

Although the details of the processes involved are not known at the present time and a comprehensive analysis is hindered by the lack of information (e.g., on ionization potentials of the selected fragments), two different ionization domains were identified within the study. The ion sputtering dominated regime for intensities of less than $1.4 \times 10^{11} \mathrm{~W} /$ $\mathrm{cm}^{2}$ and the MPI dominated region (dashed area in Figure 1) at higher intensities (Figure 3). Consequently, the wellestablished concepts and formalisms of MPI [18] were applied to the MPI dominated part of the data. Two complementary approaches were used, first being the general scaling law for the MPI, suggesting the ionization probability is taking the form [18]:

$$
P \sim \sigma_{N}^{\prime} I^{N}
$$

where $\sigma_{N}^{\prime}$ is the $N$-photon ionization cross-section and $I$ is the incident laser peak intensity. Consequently, the MPI is assumed to provide a linear dependence in a log-log plot with the slope indicating the nonlinear order. The second approach includes the concept of saturation and the ionization probability after the laser pulse is completed, $(t \rightarrow \infty)$ is [19]:

$$
P(t \rightarrow \infty)=1-e^{-\left(I / I_{\text {sat }}\right)^{2}}
$$

where

$$
I_{\text {sat }}=\hbar \omega /\left(\sigma_{N} \tau\right)^{1 / N}
$$

with $\hbar \omega$ being the photon energy and $\tau$ being the pulse duration. The $\sigma_{N}$ is the generalized $N$-photon ionization cross-section. Note that $\sigma_{N}$ corresponds to $\hbar \omega \sigma^{\prime}$. As shown in Figure 3, fitting functions (Equation 1) and (Equation 2) were applied to $\mathrm{Ag}^{+}$and $\mathrm{C}_{3} \mathrm{H}_{5} \mathrm{O}_{3}{ }^{+}$because of the amount of information available for fitting. Obviously, in the case of $\mathrm{AgOH}^{+}$and $\mathrm{C}_{3} \mathrm{H}_{5}^{+}$, the fast MPI-induced rise of the ion yield is represented by two to three points only, whereas $\mathrm{C}_{4} \mathrm{H}_{5} \mathrm{O}_{4}{ }^{+}$nontrivial dependence resulting from the ionization/fragmentation interplay provides no reliable fitting. Taking the ionization potential of $\mathrm{Ag}(7.57 \mathrm{eV})$, eight photons would be required in the standard MPI model for our photon energy of $1 \mathrm{eV}$. Interestingly, the fitting procedure provides a number of three to four photons and, therefore, the standard MPI model might not be appropriate for the direct ionization at the surface where other effects like field enhancement and symmetry breaking might play a role. An alternative explanation might be that the laser preionization accounts for $50 \%$ of the energy required for the ionization, whereas the rest is provided by the ion sputtering process, an idea supported by a mere 2-fold increase of the $\mathrm{Ag}^{+}$ion signal (Figure 3). Additional care must be taken when assessing the saturation intensities obtained from fitting the data by Equation 2 and evaluated as $2.4 \times 10^{13} \mathrm{~W} / \mathrm{cm}^{2}$ for $\mathrm{Ag}$ and $1.4 \times 10^{12} \mathrm{~W} / \mathrm{cm}^{2}$ for $\mathrm{C}_{3} \mathrm{H}_{5} \mathrm{O}_{3}$. The value of $2.4 \times 10^{13} \mathrm{~W} / \mathrm{cm}^{2}$ obtained for $\mathrm{Ag}$ is close to the one reported for postionization [13]. However note, that there is a clear experimental evidence for saturation in the data (Figure 3) already at intensities of the order of 1.7-1.9 $10^{11} \mathrm{~W} / \mathrm{cm}^{2}$. The discrepancy is explained by the lack of data points at high intensities, where the saturation takes place and, consequently, Equation 2 fits the slope resulting into $I_{\text {sat }}$ being effectively "projected" to higher values. Present modeling provided clues on the role of MPI at high intensities, but a more accurate model would be needed in order to reliably fit the experimental data.

\section{Conclusions}

The combination of mass spectrometry and laser preionization was discussed as a potential tool to observe a different variety of fragments from chemical species under analysis. The preionization-induced secondary ions in the range of 170-200 u correspond to fragments $\mathrm{C}_{\mathrm{x}} \mathrm{H}_{\mathrm{y}} \mathrm{O}_{\mathrm{z}}$ and/or $\mathrm{C}_{\mathrm{x}} \mathrm{H}_{\mathrm{y}}$ associated with $\mathrm{Ag}^{+}$as $\mathrm{C}_{\mathrm{x}} \mathrm{H}_{\mathrm{y}} \mathrm{O}_{\mathrm{z}} \mathrm{A}_{\mathrm{g}}{ }^{+} / \mathrm{C}_{\mathrm{x}} \mathrm{H}_{\mathrm{y}} \mathrm{Ag}^{+}$. Two ionization domains were identified, with the dominant ion sputtering for intensities of less than $1.4 \times 10^{11} \mathrm{~W} / \mathrm{cm}^{2}$ and dominant MPI at higher intensities. The saturation intensities obtained from fitting the data were $2.4 \times 10^{13} \mathrm{~W} / \mathrm{cm}^{2}$ for $\mathrm{Ag}$ and $1.4 \times 10^{12} \mathrm{~W} / \mathrm{cm}^{2}$ for $\mathrm{C}_{3} \mathrm{H}_{5} \mathrm{O}_{3}$, where the value for $\mathrm{Ag}$ is close to the one reported for postionization. Modest intensities of the order of $10^{11} \mathrm{~W} / \mathrm{cm}^{2}$ were already sufficient to observe significant changes in the mass spectra. Proposed approach might, therefore, eliminate the need for high peak power/high intensity laser source as used for standard postionization. A high repetition rate, compact, $100 \mathrm{~nJ}$ class fiber-based laser oscillator/amplifier can be employed with the advantage of reduced complexity/cost and improved stability. Another significant advantage is the area of the sample affected by the laser beam. The secondary neutrals in postionization are only ionized within a limited volume in the vicinity of the beam focus defined by the beam waist size and Rayleigh length. On the other hand, preionization effectively covers much larger areas of the sample, since intensities twoorders of magnitude lower are required to observe the effect. The results provided a proof of principle, while a model experiment with carefully controlled conditions is currently underway. The observed correlations of SIMS and laser preionization were shown to 
be the outcome of the mutual interplay as opposed to simple sputtering, laser irradiation, or thermal effect.

\section{Acknowledgments}

The authors gratefully acknowledge financial support by projects ERDF OP R\&D, meta-QUTE-Centrum excelentnosti kvantových technológií, APVV-0491-07 and VEGA 1/ $0522 / 10$, VEGA $1 / 0437 / 10$. The authors thank Alexei Podshivalov for the recent upgrade of the femtosecond system as well as for the long-term technical support and assistance.

\section{References}

1. Benninghoven, A.: Chemical analysis of inorganic and organic surfaces and thin films by static time-of-flight secondary ion mass spectrometry (TOF-SIMS). Angew. Chem. Int. Ed. 33, 1023-1043 (1994)

2. Van Vaeck, L., Adriaens, A., Gijbels, R.: Static secondary ion mass spectrometry: (S-SIMS) Part 1. Methodology and structural interpretation. Mass Spectrom. Rev. 18, 1-47 (1999)

3. Adriaens, A., VanVaeck, L., Adams, F.: Static secondary ion mass spectrometry (S-SIMS) Part 2: Material science applications. Mass Spectrom. Rev. 18, 48-81 (1999)

4. Vickerman, J.C., Briggs, D.: TOF-SIMS: Surface Analysis by Mass Spectrometry. IM Publications, Charlton (2001)

5. Becker, C.H., Gillen, K.T.: Surface analysis by nonresonant multiphoton ionization of desorbed or sputtered species. Anal. Chem. 56, 16711674 (1984)

6. Brummel, C.L., Willey, K.F., Vickerman, J.C., Winograd, N.: Ion beam induced desorption with postionization using high repetition femtosecond lasers. Int. J. Mass Spectrom. 143, 257-270 (1995)

7. Nicolussi, G.K., Pellin, M.J., Lykke, K.R., Trevor, J.L., Mencer, D.E., Davis, A.M.: Surface analysis by SNMS: femtosecond laser postionization of sputtered and laser desorbed atoms. Surf. Interface Anal. 24, 363-370 (1996)
8. Wucher, A., Heinrich, R., Braun, R.M., Willey, K.F., Winograd, N.: Vacuum ultraviolet single photon versus femtosecond multiphoton ionization of sputtered germanium clusters. Rapid Commun. Mass Spectrom. 12, 1241-1245 (1998)

9. He, C., Becker, C.H.: Uniform surface elemental analysis using sputtering and photoionization mass spectrometry with 6 ns pulse length Nd:YAG laser. J. Appl. Phys. 86, 3463-3468 (1999)

10. Kollmer, F., Bourdos, N., Kamischke, R., Benninghove, A.: Nonresonant laser-SNMS and TOF-SIMS analysis of sub- $\mu \mathrm{m}$ structures. Appl. Surf. Sci. 203, 238-243 (2003)

11. King, B.V., Pellin, M.J., Moore, J.F., Veryovkin, I.V., Savina, M.R., Tripa, C.E.: Estimation of useful yield in surface analysis using single photon ionization. Appl. Surf. Sci. 203-204, 244-247 (2003)

12. Vering, G., Crone, C., Kathers, P., Bijma, J., Arlinghaus, H.F.: Resonant laser-SNMS of boron for analysis of paleoceanographic samples. Appl. Surf. Sci. 252, 7163-7166 (2006)

13. Mibuka, R., Hassaballa, S., Uchino, K., Yurimoto, H., Todokoro, R., Kumondai, K., Ishihara, M.: Characteristics of post-ionization using a femto-second laser. Appl. Surf. Sci. 255, 1595-1598 (2008)

14. Willingham, D., Kucher, A., Winograd, N.: Strong-field ionization of sputtered molecules for biomolecular imaging. Chem. Phys. Lett. 468, 264-269 (2009)

15. Stump, M.J., Fleming, R.C., Gong, W.-H., Jaber, A.J., Jones, J.J., Surber, C.W., Wilkins, C.L.: Matrix-assisted laser desorption mass spectrometry. Appl. Spectrosc. Rev. 37, 275-303 (2002)

16. Mahoney, C.M., Fahey, A.J., Gillen, G.: Temperature-controlled depth profiling of poly(methyl methacrylate) using cluster secondary ion mass spectrometry. 1. Investigation of depth profile characteristics. Anal. Chem. 79, 828-836 (2007)

17. Mahoney, C.M., Fahey, A.J., Gillen, G., Xu, C., Batteas, J.D.: Temperature-controlled depth profiling of poly(methyl methacrylate) using cluster secondary ion mass spectrometry. 2. Investigation of sputter-induced topography, chemical damage, and depolymerization effects. Anal. Chem. 79, 837-845 (2007)

18. Lambropoulos, P., Tang, X.: Multiple excitation and ionization of atoms by strong lasers. J. Opt. Soc. Am. B 4, 821-832 (1987)

19. Witzel, B., Uiterwaal, C.J.G.J., Schroder, H., Charalambidis, D., Kompa, K.L.: Analysis of multiphoton ionization of metal atoms in the saturation regime using subpicosecond KrF laser pulses. Phys. Rev. A 58, 3836-3848 (1998) 